\title{
ARTICLE
}

\section{Stable Superwetting Surface Prepared with Tilted Silicon Nanowires}

\author{
Xiangman Meng ${ }^{1} \cdot$ Ailin Zhou $^{1} \cdot$ Bo Wang $^{1} \cdot$ Yu Chen $^{1} \cdot$ Yun-Hui Tang ${ }^{1} \cdot$ Hui Yan $^{1}$
}

Received: 12 May 2016/ Accepted: 8 June 2016/Published online: 6 July 2016

(C) The Author(s) 2016. This article is published with open access at Springerlink.com

\begin{abstract}
Large-scale uniform nanostructured surface with superwettability is crucial in both fundamental research and engineering applications. A facile and controllable approach was employed to fabricate a superwetting tilted silicon nanowires (TSNWs) surface through metal-assisted chemical etching and modification with low-surface-energy material. The contact angle (CA) measurements of the nanostructured surface show a large range from the superhydrophilicity (the CA approximate to $0^{\circ}$ ) to superhydrophobicity (the CA up to $160^{\circ}$ ). The surface becomes antiadhesion to water upon nanostructuring with a measured sliding angle $(\alpha)$ close to $0^{\circ}$. Moreover, the fluorinated TSNWs surface exhibits excellent stability and durability because strong chemical bonding has been formed on the surface.
\end{abstract}

\section{Graphical Abstract}

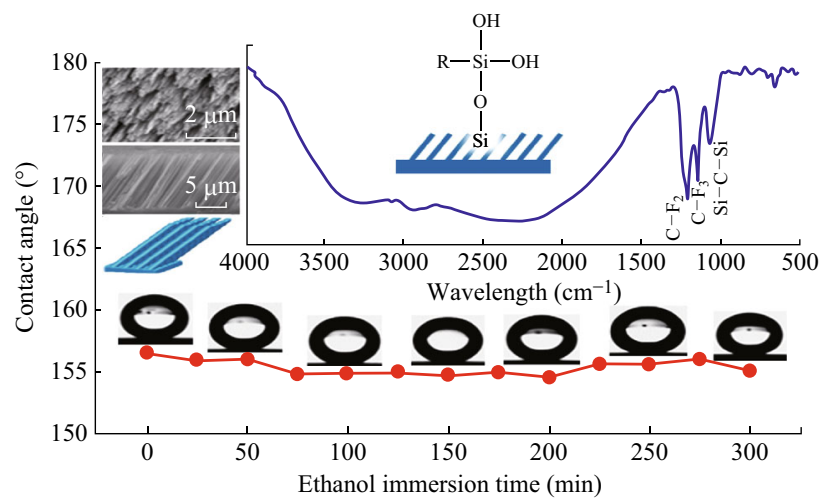

Keywords Tilted silicon nanowires $\cdot$ Chemical etching $\cdot$ Superwettability $\cdot$ Stability

Bo Wang

wangbo@bjut.edu.cn

$1 \quad$ School of Materials Science and Engineering, Beijing University of Technology, Beijing 100124, People's Republic of China

\section{Introduction}

Superwetting surface with drop sliding angle $(\alpha)$ less than $5^{\circ}$ and water contact angles (CA) lower than $5^{\circ}$ or higher than $150^{\circ}$ has drawn much more attention in recent years because of the limited contact angle and rollability $[1,2]$. The superwetting properties of solid surface has crucial applications in our daily life as well as engineering fields, 
such as self-cleaning, digital microfluidic devices, biomedical engineering, and silicon hybrid solar cells [3-7]. There are two ways to obtain the superwetting properties of existing artificial surface. One is to fabricate special rough geometric microstructures on the surface, which is a successful technique to control over the wettability of solid $[8,9]$, the other is to modify a rough surface with low-surface-energy chemical composition $[10,11]$. Recently, numerous approaches have been explored to fabricate solid surface with superwetting property, such as self-assembly, lithography selective etching of solid substrates and chemical etching [12-14], etc. However, there are still some existing limitations in the fabrication of large-scale superwetting surface and practical applications because of low stability and durability, costly and complicated fabrication procedures. Furthermore, most of the superhydrophobic surfaces have pretty high CA, chemical or geometrical heterogeneous surface which usually exhibits contact angle hysteresis and droplets adhere to the surface, so that they are greatly restricted to the applications [15].

In this study, tilted silicon nanowires (TSNWs) have been fabricated on the surface of Si subtracts by metalassisted chemical etching (MaCE) method. The surface with TSNWs modified by material of low surface energy could greatly expand the flexible application in designing of the silicon nanowires (SiNWs) devices and may introduce new characteristics [16]. Special features of the nanostructure include a microscale smooth surface with superwetting property, antiadhesion to water, and a large range from superhydrophilicity to superhydrophobicity. Systematical investigations ranging from structural characterizations and surface wettability studies on the analysis of the mechanism have been reported. The studies are further probed into the orientation etching of SiNWs and the wettability of the silicon surface prepared with TSNWs through modified hydrophobic agent.

\section{Experiment}

\subsection{Substrate Preparation}

In our experiments, $\mathrm{Si}$ (110) wafers were used as etching substrates. The commercial $\mathrm{Si}$ wafers were cut into $2 \times 1$ $\times 0.1 \mathrm{~cm}^{3}$ and ultrasonically cleaned with toluene, acetone, ethanol, and deionized water (DI-water) for several cycles to entirely remove organics. To totally remove the residual metal particles of silicon surface, the cleaned $\mathrm{Si}$ wafers were immersed in $\mathrm{H}_{2} \mathrm{SO}_{4}(97 \%)$ and $\mathrm{H}_{2} \mathrm{O}_{2}(40 \%)$ oxidant solution (piranha solution) in a volume ratio 3:1 for $10 \mathrm{~min}$ at room temperature, followed by $1 \mathrm{~min}$ DI-water rinse then dried by $\mathrm{N}_{2}$ gas. A thin oxide layer would be formed on the surface which presents the hydrophilicity.

\subsection{Orientation Etching and Fabrication of Superwetting Surface}

Before etching, the thin oxide layers were cleaned by $5 \%$ HF solution for $3 \mathrm{~min}$. The MaCE process consists of two basic steps. Firstly, placing the substrates into solution containing $\mathrm{HF}(4.8 \mathrm{M})$ and $\mathrm{AgNO}_{3}(0.01 \mathrm{M})$ for $30 \mathrm{~s}$ under atmosphere ambient with the purpose of silver deposition. Secondly, the Ag-loaded $\mathrm{Si}$ wafers were immersed in mixed solution with $\mathrm{HF}(4.8 \mathrm{M})$ and $\mathrm{H}_{2} \mathrm{O}_{2}(0.2 \mathrm{M})$ for $30 \mathrm{~min}$. After the MaCE process, the as-prepared samples were dipped into dilute $\mathrm{HNO}_{3}$ in a volume ratio 1:1 to dissolve the silver nanoparticles (AgNPs) and then rinsed with DIwater. The etching process was controlled at $25^{\circ} \mathrm{C}$ by water bath and the large-area TSNWs arrays were obtained. The nanostructured surface was then modified by a hydrophobic layer with $1 \mathrm{H}, 1 \mathrm{H}, 2 \mathrm{H}, 2 \mathrm{H}$-Perfluorodecyltriethoxysilane (PFOS) and performed superwettability. The nanostructured wafers were immersed in the $1 \%$ PFOS/ethanol solution to get the surface adopted with PFOS layer. To accelerate PFOS hydrolysis and condensation and form a stable hydrophobic nanostructured surface, the modified samples were dried at $60{ }^{\circ} \mathrm{C}$ for $5 \mathrm{~h}$.

\subsection{Characterizations}

The morphologies of the sample were characterized by fieldemission scanning electron microscopy (FESEM, S4800) observations at $15 \mathrm{kV}$ accelerating voltage. The TSNW lengths and angles were measured from the SEM images. The surface roughness ( $R \mathrm{a})$ was obtained by surface roughness measuring instrument (SU FCOM4800A) scanning multiple lines of $5 \times 10^{4} \mu \mathrm{m}$. The FT-FIR spectrometer (Spectrum 400) was useful for the analysis of chemical composition. To characterize the wetting properties of the nanowire arrays, the $\mathrm{CA}$ and $\alpha$ measurements of water drops were performed using the Vertion 2.8 goniometer equipped from Surface Tech Co. Ltd, the droplet volumes with $5 \mu \mathrm{L}$ water were repeated at least at five different locations on the substrate in each experiment. Self-cleaning stability was confirmed by being immersed in ethanol and outdoor test.

\section{Results and Discussion}

\subsection{SiNWs Arrays on Silicon Wafers}

The fabrication processes have been described in the above experimental section. The SEM images of as-prepared 

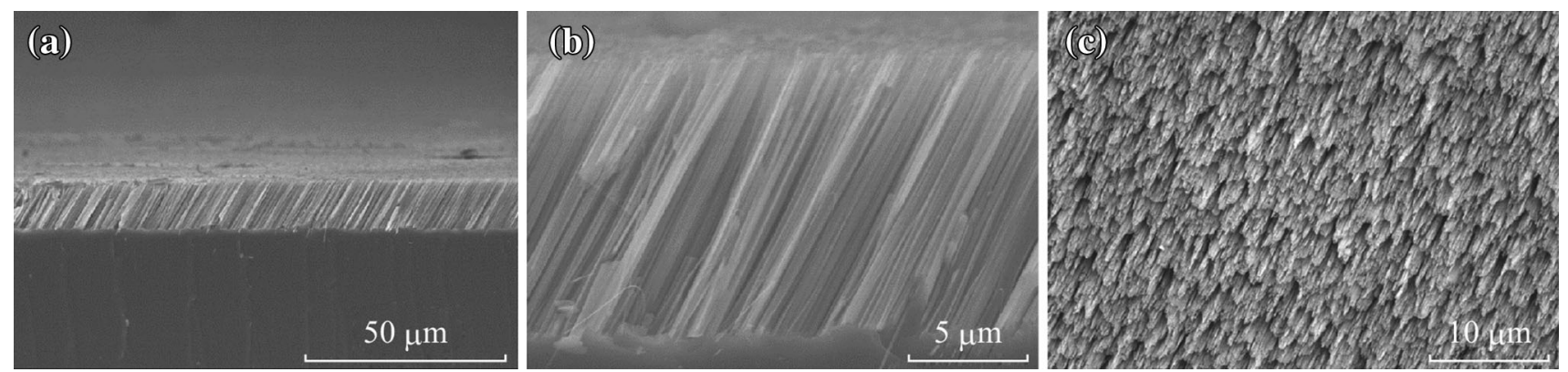

Fig. 1 SEM images of the as-prepared SiNWs arrays on Si (110) wafer. a Large-scale cross-sectional SEM image, $\mathbf{b}$ magnified cross-sectional image, and c large-scale top view

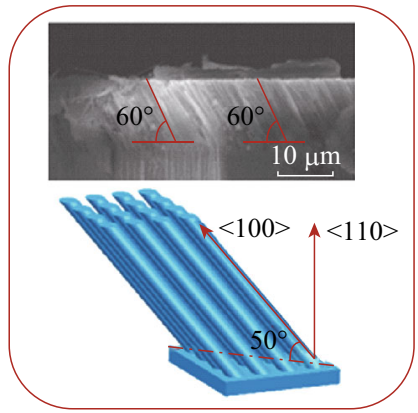

(a)

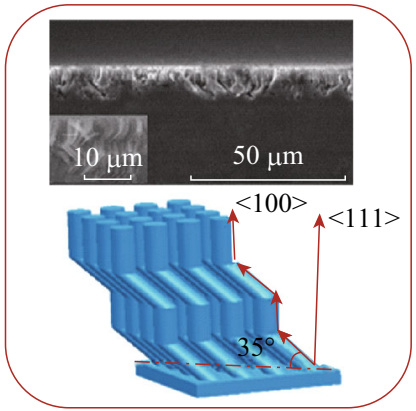

(b)

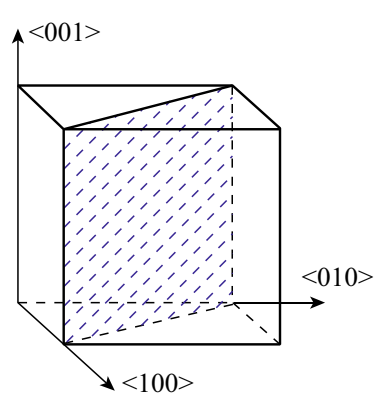

(c)

Fig. 2 Schematic evolution of SiNWs arrays on a Si-(110), and b Si-(111) wafers. c Schematic of (100) directions on silicon crystal surface

TSNWs arrays on Si-(110) wafer are shown in Fig. 1. The large-scale cross-sectional image of Fig. 1a and the top view in Fig. 1c with low magnification indicate that the SiNWs arrays obtained from $\mathrm{Si}$ (110) wafer are uniform on the entire wafer surface. Figure $1 \mathrm{~b}$ shows the detailed cross section for SiNWs under high magnification in which all SiNWs are clear, long, and uniform, the length is about $13 \mu \mathrm{m}$, and the diameter is in the range of $80-550 \mathrm{~nm}$. The TSNWs are regularly inclined to the wafer surface in both $60^{\circ}$ on two mutually perpendicular cross sections as shown in Fig. 2a, whose direction deviates from (110) plane approximately to $50^{\circ}$. The direction corresponds to the crystallographic lattices of the $\mathrm{Si}$ (100) orientations. In another words, the etching orientation on $\mathrm{Si}$ (110) wafer is always along (100) direction. The etching direction can be interpreted according to lattice configuration of oriented silicon surface and the passivation effect on $H$-terminated planes. The orientation dependence mainly depends on the anisotropic dissolution of the silicon surface. The removal rate of silicon atoms from the surface in (100) direction is far faster than the other two directions. According to backbond strength theory, the (100) plane has two bonds symmetrically directed into the reactive solution while those on the (111) plane have three, leading to a geometry that sterically prefers etching silicon atoms along the (100) direction $[17,18]$. Besides, the pretreatment of HF solution in the Experimental section essentially removed the native oxide of the silicon surface, and further passivated the surface with the formation of $\mathrm{Si}-\mathrm{H}$ terminations [19]. The $\mathrm{Si}-H$ terminations on the silicon wafer could also induce etching along (100) direction in a HF solution [20, 21].

In our experiments, one-dimensional zigzag SiNWs were obtained on $\mathrm{Si}$ (111) substrate, in agreement with existing reports (Fig. 2b) [7, 19]. Compared with the SiNWs prepared on $\mathrm{Si}$ (111), the majority of the nanowire morphologies on $\mathrm{Si}$ (110) wafers are more uniform. The reason is that there are three (100) directions on silicon crystal surface according to the crystal structure of silicon (Fig. 2c), and the favored (100) etching orientation always has the smallest angle relative to the substrate normal. They are nonidentically pointing outward from the surface to the internal of the $\mathrm{Si}$ (110) wafer, while equivalently on a Si (111) substrate (Fig. 2b). The opportunity of etching orientation along the three (100) directions are equal on $\mathrm{Si}$ (111) wafer, leading to a seemingly disordered nanostructure surface and the SiNWs congregated bundles. Whereas SiNWs can along the favored (100) direction on Si (110) wafers and the nanostructured surface shows uniformity 

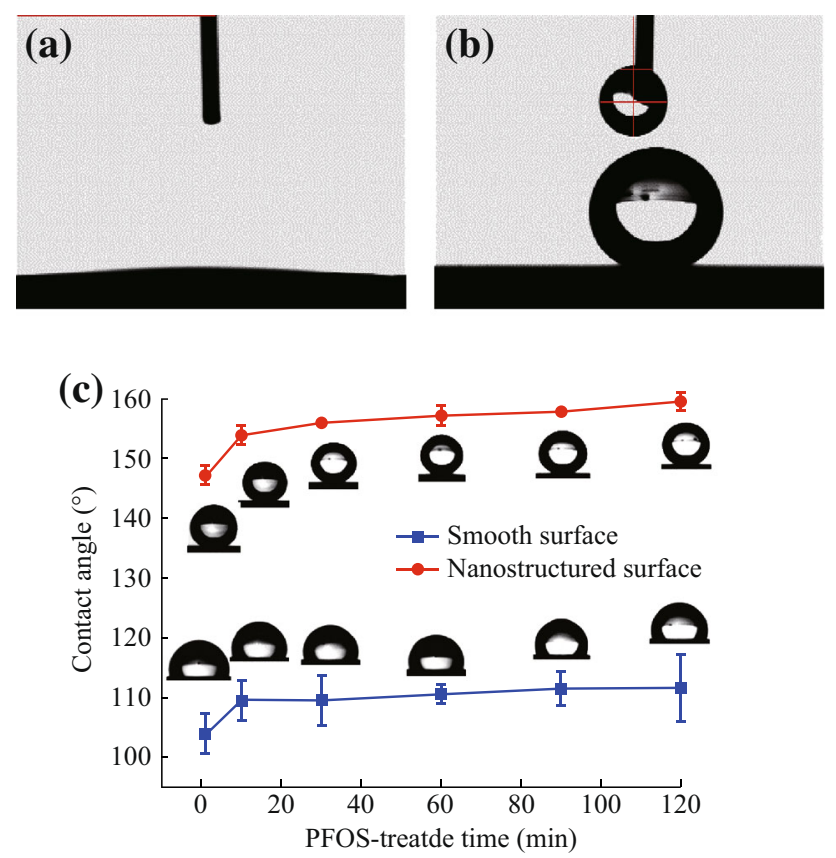

Fig. 3 Photographs of water droplets on a sample S-2, b sample S-2 treated by PFOS. c Variation in the water contact angles of the smooth and nanostructured surface which be modified by PFOS against time duration

[16]. The commercial silicon wafers possess an orientation deviation about $\pm 0.5^{\circ}$ according to GB/T12962-2005 standards, one of the two equivalent (100) directions on $\mathrm{Si}$ (110) wafer will be the favored etching direction, and thus the nanowire morphologies on $\mathrm{Si}$ (110) wafers are also uniform. Despite the variety of shapes, most SiNWs on $\mathrm{Si}$ substrate always oriented in the (100) direction. Based on the above experimental results, the schematic of SiNWs on three various oriented silicon wafer by MaCE method is shown in Fig. 2a, b.

\subsection{Wettability}

The $R \mathrm{a}$ of TSNWs surface $(0.4313 \mu \mathrm{m})$ is much higher than the nanostructured $\mathrm{Si}(100)$ wafers $(0.08220 \mu \mathrm{m})$ and $\mathrm{Si}(111)$ wafers $(0.1064 \mu \mathrm{m})$. It is well known that surface with large roughness is more conducive to the adhesion of PFOS. Thus the wettability of TSNWs surface with lowsurface-energy materials was studied. The volume of water drops was set in $5 \mu \mathrm{L}$ and the dropping height remained at $0.165 \mathrm{~mm}$ to avoid the effect of impaction. According to the Weber number, the impaction influence of a deposited drop on nanostructured surface is analyzed by $W=\rho v^{2} d l$ $\sigma$, in which $W$ represents Weber number, $\rho$ is water density, $v$ is impacting velocity, $d$ is drop diameter, and $\sigma$ is the liquid surface tension $[22,23]$. In this experiment, the relevant parameters are liquid density $\rho=998 \mathrm{~kg} \mathrm{~m}^{-3}$, the drop's impacting velocity $v=0.033 \mathrm{~m} \mathrm{~s}^{-1}$, the drop

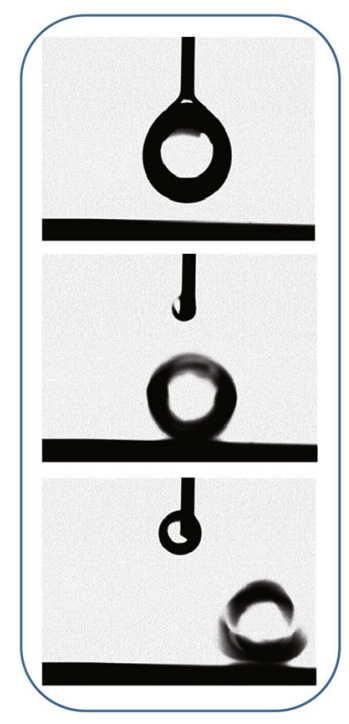

(a)

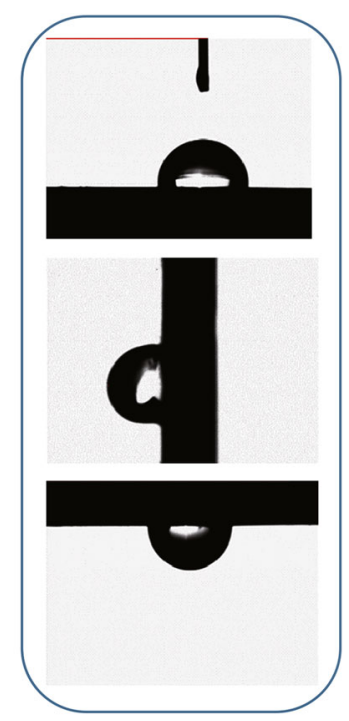

(b)
Fig. 4 Behaviors of drops on the inclined surface. a Water drops on the superhydrophobic surface. b Water drops on PFOSS-treated Si (110) wafer

diameter $d=2.6 \times 10^{-3} \mathrm{~m}$, and the liquid surface tension $\sigma=7.2 \times 10^{-3} \mathrm{~N} \mathrm{~m}^{-3}$; the Weber number was about 0.039 , illustrating that the effect of impaction is relatively small that can be ignored.

Figure 3a shows that the water droplet can nearly fully spread on the surface and the CA is less than $5^{\circ}$. The superhydrophilicity may be considered as a result of the $R$ a and formation of some polar radicals. After being immersed in $1 \%$ PFOS/ethanol solution for $30 \mathrm{~min}$, the surface turns to be superhydrophobic and the CA approximates to $160^{\circ}$ (Fig. 3b). The CA of the surface varied with the PFOS-treated time is shown in Fig. 3c. The CA of the smooth surface is $103^{\circ}$ and the as-prepared surface is $147^{\circ}$ after $1 \mathrm{~min}$. When the fluorination time prolongs to 10,30 , 60, 90, and $120 \mathrm{~min}$, the CA of the smooth surface is around $110^{\circ}$ while the as-prepared surface is all above $154^{\circ}$. The as-prepared surface exhibits excellent superhydrophobicity. The high CA is also directly attributed to the $R \mathrm{a}$, as mentioned above. The CA of the superhydrophobic surface is more uniform compared to nonstructured surface according to the standard error as shown in Fig. 3c. The PFOS is difficult to distribute evenly and agglomerates on the polished surface because of smoothness, while the TSNWs surface is rougher which enables the molecules to attach to the surface uniformly.

Drops deposited on the superhydrophobic surface and its sidling process with a fixed angle of $1.5^{\circ}$ is shown in Fig. 4a. The drop slides on the surface quite quickly within short time. The surface like the lotus leaf surface have both a large CA (higher than $150^{\circ}$ ) and a small $\alpha$ (less than $5^{\circ}$ ), also known to be antiadhesive to water, are crucial for the 


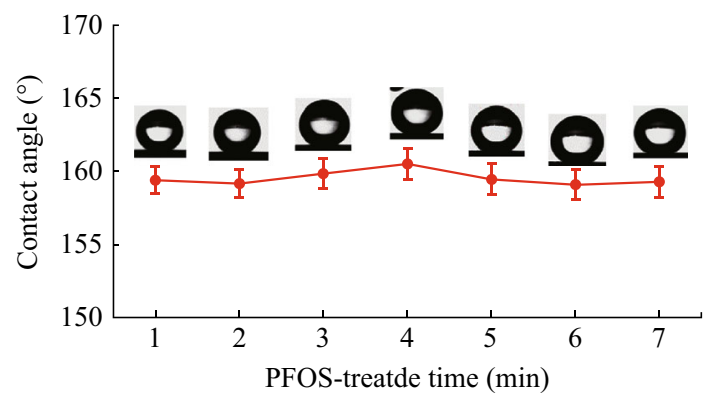

Fig. 5 Variation of the CA against air exposure time duration. Samples were collected every day and measured for CA

superwetting surface [24]. The $\alpha$ on the lotus-like TSNWs surface was much lower than that on polished wafers with no nanostructures. Water droplets adhere to the smooth surface even when the wafer is tilted vertically (the pinning position, Fig. 4b, middle) or inverted (Fig. 4b, bottle). Water droplets on the water repellent surface favor rolling like an elastic ball, leaving the surface completely and keep dry or even take away the contaminants on the surface $[25,26]$.

Wetting stability of the Superhydrophobic nanostructured surface was also extensively studied. Figure 5 shows the CA of samples which exposed to air for 7 days, measured every day. The superhydrophobicity seems to contribute to keeping the surface clean, it shows an almost flat CA trend over a period of days. It illustrates that the PFOS attached to the silicon surface firmly and steadily. Despite the fog and haze on third days and fourth days, the CA of surface is still approximately at $159^{\circ}$. According to the Arkles theory [27], the hydrolysis reaction of siloxane has been formed once PFOS contacts with silicon. Through the dehydration reaction of the fluorine-bearing organosilane molecules with hydroxyl groups of SiNWs, the highly polar hydroxyl of silicon surface converts to a low polarity ether bond. Eventually, PFOS is covalently attached onto the nanostructured silicon surface and form interfacial region. Silicon surface is covered by hydrophobic film with $-\mathrm{CF}_{2}$ and $-\mathrm{CF}_{3}$ group. The surface energy of the silicon is

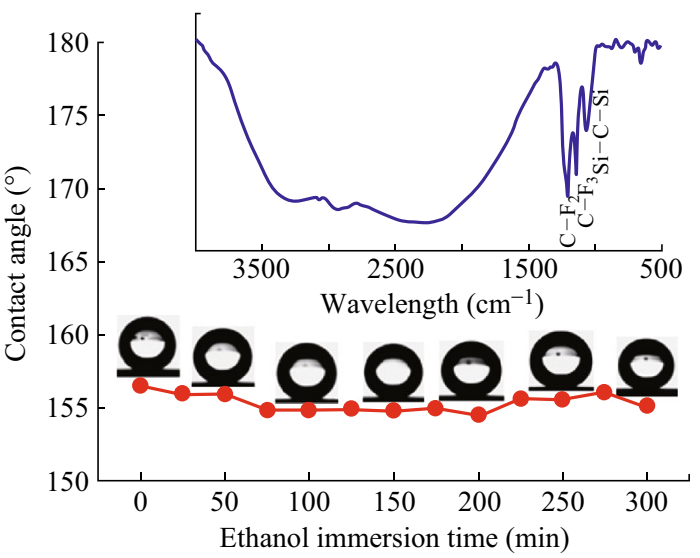

Fig. 7 Variation of the CA against ethanol immersion time duration. The inset is FTIR spectrum of the superhydrophobic surface after immersed in ethanol for $300 \mathrm{~min}$

obviously reduced and the CA has improved. The specific reaction process is shown in Fig. 6.

After being in ethanol for a period of time, the surface shows less decrease in contact angle (Fig. 7). The inset (FTIR spectrum) shows that silane groups still exist on the silicon surface after 30 min immersion. The absorption constant at 1148 and $1200 \mathrm{~cm}^{-1}$ are corresponded to symmetric stretching vibration modes of $-\mathrm{CF}_{3}$ and asymmetric stretching vibration modes of $-\mathrm{CF}_{2}$, respectively. $\mathrm{Si}-$ $\mathrm{O}-\mathrm{Si}$ asymmetric stretching vibration bands occurred at $1075 \mathrm{~cm}^{-1}$ [28]. It is proved that the dependence of the PFOS on the nanostructured surface is a chemical bonding. This is consistent with the above-mentioned Arkles theory.

\section{Conclusions}

Fabrication of TSNWs arrays with extremely uniform top surface on silicon substrates by MaCE approach has been reported in this article. The etching orientation on $\mathrm{Si}$ wafers<smiles>CO[Si](C)(OC)OC</smiles><smiles>[R][Si](O)(O)OC1[C@@H](C)[13CH2][C@@H]1C</smiles><smiles>[R][Si](O)(O)O[Si](C#C)[Si]C1CC1</smiles>

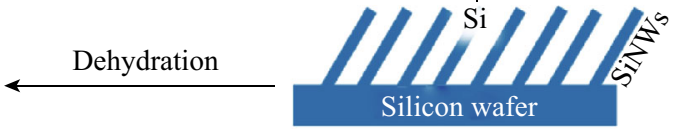

Fig. 6 Schematic illustration of the reaction between PFOS and the substrate 
always follows the (100) direction. Changing the surface structure and surface chemical modification, the wetting performance of nanostructured surface can be controlled from hydrophilicity to superhydrophilic or superhydrophobicity, from adhesion with water to antiadhesion to water. The above experiments prove that the wettability of silicon surface can be evidently enhanced via chemical etching and PFOS treatments. The wetting property of superhydrophobic film is very stable with chemical bond between PFOS and substrate, which can be potentially useful for super extensive applications, including antifouling, digital microfluidic devices, and nonwetting liquid transfer.

Open Access This article is distributed under the terms of the Creative Commons Attribution 4.0 International License (http://crea tivecommons.org/licenses/by/4.0/), which permits unrestricted use, distribution, and reproduction in any medium, provided you give appropriate credit to the original author(s) and the source, provide a link to the Creative Commons license, and indicate if changes were made.

\section{References}

1. J. Zhang, S. Seeger, Polyester materials with superwetting silicone nanofi laments for oil/water separation and selective oil absorption. Adv. Funct. Mater. 21(24), 4699-4704 (2011). doi:10.1002/adfm.201101090

2. P. Zhang, S. Wang, S. Wang, L. Jiang, Superwetting surfaces under different media: effects of surface topography on wettability. Small 11(16), 1939-1946 (2015). doi:10.1002/smll. 201401869

3. J. Drelich, E. Chibowski, D.D. Meng, K. Terpilowski, Hydrophilic and superhydrophilic surfaces and materials. Soft Matter 7(21), 9804-9828 (2011). doi:10.1039/c1sm05849e

4. Y. Huang, D.K. Sarkar, X. Chen, Fabrication of superhydrophobic surfaces on aluminum alloy via electrodeposition of copper followed by electrochemical modification. Nano-Micro Lett. 3(3), 160-165 (2011). doi:10.3786/nml.v3i3.p160-165

5. B. Xin, J. Hao, Reversibly switchable wettability. Chem. Soc. Rev. 39(2), 769-782 (2010). doi:10.1039/B913622C

6. Y. Zhang, W. Cui, Y. Zhu, F. Zu, L. Liao, S.T. Lee, B. Sun, High efficiency hybrid PEDOT:PSS/nanostructured silicon Schottky junction solar cells by doping-free rear contact. Energ. Environ. Sci. 8(1), 297-302 (2015). doi:10.1039/C4EE02282C

7. R. Ghosh, P.K. Giri, Efficient visible light photocatalysis and tunable photoluminescence from orientation controlled mesoporous Si nanowires. RSC Adv. 6(42), 35365-35377 (2016). doi:10.1039/C6RA05339D

8. Y. Lai, F. Pan, C. Xu, H. Fuchs, L. Chi, In situ surface-modification-induced superhydrophobic patterns with reversible wettability and adhesion. Adv. Mater. 25(12), 1682-1868 (2013). doi:10.1002/adma.201203797

9. T. Darmanin, F. Guittard, Wettability of conducting polymers: from superhydrophilicity to superoleophobicity. Prog. Polym. Sci. 39(4), 656-682 (2014). doi:10.1016/j.progpolymsci.2013.10. 003

10. Y. Coffinier, G. Piret, M.R. Das, R. Boukherroub, Effect of surface roughness and chemical composition on the wetting properties of silicon-based substrates. C. R. Chim. 16(1), 65-72 (2013). doi:10.1016/j.crci.2012.08.011
11. L. Yin, Y. Wang, J. Ding, Q. Wang, Q. Chen, Water condensation on superhydrophobic aluminum surfaces with different low-surface-energy coatings. Appl. Surf. Sci. 258(8), 4063-4068 (2012). doi:10.1016/j.apsusc.2011.12.100

12. E. Miele, M. Malerba, M. Dipalo, E. Rondanina, A. Toma, F.D. Angelis, Controlling wetting and self-assembly dynamics by tailored hydrophobic and oleophobic surfaces. Adv. Mater. 26(24), 4179-4183 (2014). doi:10.1002/adma.201400310

13. X. Zhang, J. Zhang, Z. Ren, X. Li, X. Zhang, D. Zhu, T. Wang, T. Tian, B. Yang, Morphology and wettability control of silicon cone arrays using colloidal lithography. Langmuir 25(13), 7375-7382 (2009). doi:10.1021/la900258e

14. H. Bellanger, T. Darmanin, E. Taffin de Givenchy, F. Guittard, Chemical and physical pathways for the preparation of superoleophobic surfaces and related wetting theories. Chem. Rev. 114(5), 2694-2716 (2014). doi:10.1021/cr400169m

15. L. Gao, T.J. McCarthy, Contact angle hysteresis explained. Langmuir 22(14), 6234-6237 (2006). doi:10.1021/la060254j

16. J. Ma, L. Wen, Z. Dong, T. Zhang, S. Wang, L. Jiang, Aligned silicon nanowires with fine-tunable tilting angles by metal-assisted chemical etching on off-cut wafers. Phys. Status Solidi R 7(9), 655-658 (2013). doi:10.1002/pssr.201307190

17. R. Smith, S. Collins, Porous silicon formation mechanisms. J. Appl. Phys. 71(8), R1 (1992). doi:10.1063/1.350839

18. V. Lehmann, The physics of macropore formation in low doped n-type silicon. J. Electrochem. Soc. 140(10), 2836-2843 (1993). doi:10.1149/1.2220919

19. C.Y. Chen, C.P. Wong, Unveiling the shape-diversified silicon nanowires made by $\mathrm{HF} / \mathrm{HNO}_{3}$ isotropic etching with the assistance of silver. Nanoscale 7(3), 1216-1223 (2015). doi:10.1039/ C4NR05949B

20. M. Christophersen, J. Carstensen, S. Rönnebeck, C. Jäger, W. Jäger, H. Föll, Crystal orientation dependence and anisotropic properties of macropore formation of $\mathrm{p}$ - and n-type silicon. J. Electrochem. Soc. 148(6), E267-E275 (2001). doi:10.1149/1. 1369378

21. M. Christophersen, J. Carstensen, A. Feuerhake, H. Föll, Crystal orientation and electrolyte dependence for macropore nucleation and stable growth on p-type Si. Mater. Sci. Eng. B 69-70, 194-198 (2000). doi:10.1016/S0921-5107(99)00262-7

22. D. Quéré, Leidenfrost dynamics. Annual Rev. Fluid Mech. 45(1), 197-215 (2013). doi:10.1146/annurev-fluid-011212-140709

23. P. Tsai, M.H.W. Hendrix, R.R.M. Dijkstra, L. Shui, D. Lohse, Microscopic structure influencing macroscopic splash at high Weber number. Soft Matter 7(24), 11325-11333 (2011). doi:10. 1039/c1sm05801k

24. S. Li, Y. Li, H. Li, L. Zhang, J. Zhai et al., Super-hydrophobic surfaces: from natural to artificial. Adv. Mater. 14(24), 1857-1860 (2002). doi:10.1002/adma.200290020

25. B. Zhang, Y. Li, B. Hou, One-step electrodeposition fabrication of a superhydrophobic surface on an aluminum substrate with enhanced self-cleaning and anticorrosion properties. RSC Adv. 5(121), 100000-100010 (2015). doi:10.1039/C5RA21525K

26. T.M. Schutzius, S. Jung, T. Maitra, G. Graeber, M. Kohme, D. Poulikakos, Spontaneous droplet trampolining on rigid superhydrophobic surfaces. Nature 527(7576), 82-85 (2015). doi:10. 1038/nature 15738

27. J. Gu, P. Xiao, Y. Huang, J. Zhang, T. Chen, Controlled functionalization of carbon nanotubes as superhydrophobic material for adjustable oil/water separation. J. Mater. Chem. A 3(8), 4124-4128 (2015). doi:10.1039/C4TA07173E

28. H.J. Jeong, D.K. Kim, S.B. Lee, S.H. Kwon, K. Kadono, Preparation of water-repellent glass by sol-gel process using perfluoroalkylsilane and tetraethoxysilane. J. Colloid Interf. Sci. 235(1), 130-134 (2001). doi:10.1006/jcis.2000.7313 